\title{
The Radiation, Interplanetary Shocks, and Coronal Sources (RISCS) Toolset
}

G.P. Zank(1,2), and J. Spann(3) for the RISCS Collaboration

(1) Center for Space Plasma and Aeronomic Research (CSPAR), University of Alabama in Huntsville, Huntsville, AL 35805

(2) Physics Department, University of Alabama in Huntsville, Huntsville, AL 35899

(3) NASA, Marshall Space Flight Center, MSFC, Huntsville, AL 35812

We outline a plan to develop a physics based predictive toolset RISCS to describe the interplanetary energetic particle and radiation environment throughout the inner heliosphere, including at the Earth. To forecast and "nowcast" the radiation environment requires the fusing of three components: 1) the ability to provide probabilities for incipient solar activity; 2) the use of these probabilities and daily coronal and solar wind observations to model the 3D spatial and temporal heliosphere, including magnetic field structure and transients, within $10 \mathrm{AU}$; and 3) the ability to model the acceleration and transport of energetic particles based on current and anticipated coronal and heliospheric conditions. We describe how to address 1) - 3) based on our existing, well developed, and validated codes and models. The goal of RISCS toolset is to provide an operational forecast and "nowcast" capability that will a) predict solar energetic particle (SEP) intensities; b) spectra for protons and heavy ions; c) predict maximum energies and their duration; d) SEP composition; e) cosmic ray intensities, and f) plasma parameters, including shock arrival times, strength and obliquity at any given heliospheric location and time. The toolset would have a 72 hour predicative capability, with associated probabilistic bounds, that would be updated hourly thereafter to improve the predicted event(s) and reduce the associated probability bounds. The RISCS toolset would be highly adaptable and portable, capable of running on a variety of platforms to accommodate various operational needs and requirements. 